\title{
Virgil, Robot for Museum Experience: \\ Study on the opportunity given by robot capability to integrate the actual museum visit.
}

\author{
Luca Giuliano, Miguel Efrain Kaouk Ng, Maria Luce Lupetti, Claudio Germak \\ Politecnico di Torino \\ Torino, Italy \\ \{luca.giuliano, miguel.kaoukng, maria.lupetti, claudio.germak\}@polito.it
}

\begin{abstract}
Robotics platforms are becoming more and more present in people everyday life. A reflection on multimedia technologies that are currently used in the museum experiences has been made. In this paper, we present and describe a robotic system, called "Virgil" and a remote tele-operation application used as a support instrument for the museum guide in order to enhance the museum experience and increase the cultural value of the territorial heritage. The application is based on a Cloud Robotic infrastructure that contains ROS nodes and exposes a set of APIs to the user.
\end{abstract}

Keywords: Cloud Robotics Platform; Human Robotic Interaction; Robot mobile Telepresence; Intelligence Robots.

\section{INTRODUCTION}

Nowadays the technology development has facilitated the evolution of Robotics Service Applications. Due to our cultural background we have always linked the robotics applications to the industrial production. Present-day life reality, instead, shows up a world that is slowly getting populated by highly intelligent technological systems. Therefore humans are increasingly getting more and more used to share physical and digital spaces with robotic platforms [1]. Taking into account this scenario, the research area of cultural heritage, assume a very high importance and become very interesting. Thanks to technological developments in recent decades it has been possible to proof concepts with service robotic applications that are able to offer an added value to the museum experience [2], and complement the current technologies used (Audio guides, Multimedia guides and Personal technologies). Our aim is to present and describe an integrated solution of a service robotic application that is based on a Cloud Robotic Platform, and use a mobile robot, called "Virgil", as a support element to complement and improve the museum experience at the Racconigi's castle inserted in the Cultural Heritage of the lands of the Savoy.

\section{SYSTEMS AND APPLICATIONS IN MUSEUM SPACES. STATE OF THE ART AND DESIGN CUES}

The main objective of a museum is to preserve the artistic heritage and to become a mediator between cultural heritage and visitors. The museum guide who has the task to protect the historical and territorial cultural memory plays a key role of this relationship. An accurate and detailed environment description creates an empathic connection between structure and the people. In order to create an empathic connection it is necessary to put the museum guide in the condition to satisfy the following requirements [3]:

- Activate a stream of consciousness in the visitor through a sensorial approach.
- Increase the visitor sense of exploration.

- Increase the degrees of freedom of the visitor.

The first phase of our research, has been the analysis of the actually used technology in the museum context. This analysis has allowed us to understand the attractive and functional aspects of the multimedia activities and define two main categories: on site applications (interaction inside the museum) and on remote applications (interaction from remote). The main disadvantages of the remote application are the loss of contact with the cultural territory in which the museum is located (unlinking territory and museum could impoverish the museum cultural message). On the other side, there is the image digitization, which main advantage is the sharing of the museum information making them accessible to everybody through the online fruition. Moreover, museum experience requires a continuous updating. Rotation of artworks exposition and modification of open spaces to the public on visit is a usual action to enhance the attractiveness of the museum experience. Furthermore, some space are forced to be temporarily or permanently closed because of the safety of spaces restoration. Although most technologies solutions are very evolved, there are not still enough flexible to solve this particular aspect.

This work considers the possibility of using a robot as a support instrument for the museum guide in order to enhance the museum experience and integrate aspects from the two different main categories. With this intent a set of design guidelines useful for the concept development of "Virgil project" were formulated:

- Our robot should not compete with the work of the museum guide or replace it.

- Our solution has not to ruin the environment and the artworks of the place where it operates.

- The system has to make possible the exploration of inaccessible places providing a more immersive and complete experience to the visitors.

- The mobile robot platform has to be updatable and integrable to several context of use.

- Our solution has not to be a separated element respect to the territorial system where it operates.

\section{FRAMEWORK DESCRIPTION}

The proposed system (Fig. 1) is based on the concept of Cloud Robotics [4]. Our cloud robotics platform is an infrastructure that makes use of a cloud architecture aiming at create a more powerful and centralized brain, where the robot become a cloud agent who relies most of their computational load and data storage on remote servers. Moreover, our cloud robotics 
platform is also able to guarantee the robustness needed for long-term operativeness in the robotics applications and to expose simple APIs to the final user [5], [6]. In our framework a four wheels drives mobile robot able to move through indoor structured environments, is deployed and communicate with the Cloud Robotics Platform (CRP) through Long Term Evolution (LTE) technology. CRP has a central unit called "Robot Clone Manager" which takes care of all the jobs necessary to communicate user with robot. Basically, RCM manages the information from the robot and sensors and exposes them to the user. At the same time, receives commands from the user, processes and transmits them to the robot. Finally, the user is able to interact with the robot through a Graphical User Interface (GUI), displayed on a PC or on smart devices via a web browser.

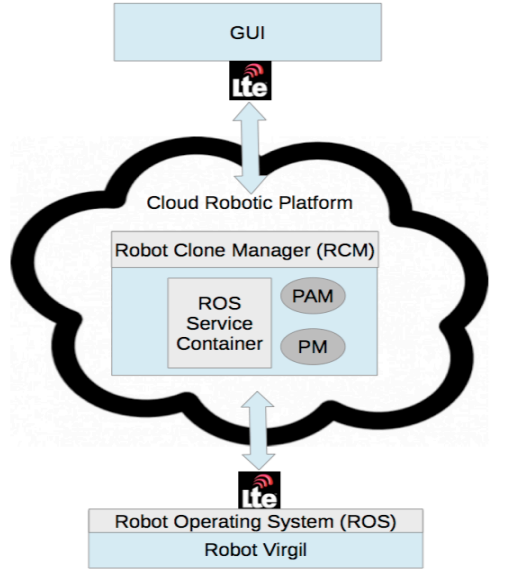

Fig 1. Schematic description of the framework used.

CRP is based on the Robotics Operating System framework (ROS), in order to exploit the vast amount of already available ROS nodes and abstract robot hardware towards ROS service logic applications.

Robot Clone Manager (RCM) is the element able to generate a Service Container (SC), which hosts all ROS nodes necessary to control the robot. These nodes can perform computation, in order to support abstraction of hardware layer (robot and sensors) towards applications on top of the cloud robotics platform. As a result, there is a virtualization of the physical robot and a Robot clone (cloud-agent) is created. This clone resides on remote servers, where it can load and store large amounts of data and information. RCM is also supported by a Platform Manager (PM) and the platform API manager (PAM). PM is the element in charge of manages the objects inside RCM. It main functions are sending and receiving commands to manipulate objects, listen to and create events to notified the objects state changes. PAM instead is an object that exposes to the user a set of APIs (REST, JS) for platform management (commands and events).

\section{TElePRESENCE SERVICE APPLiCATION}

The service application developed is composed by: a Hardware Layer that is in charge of abstract the HW of the system, a Service Layer which contain the ROS node and an Application Layer that hold the interaction elements with the user. The scenario that we have considered is a tele-presence robot that is tele-operated by the museum guide to explore temporary inaccessible areas of the Racconigi's castle. The real time video streaming (sending by the camera) is shown to the visitors on devices, such as screens and personal devices. In our application, the software has been developed using open source technologies. In particular, we have used Robot Operating System (ROS) in $\mathrm{C}++$ under Linux. The latency of the application for the transmission and execution commands is less than $200 \mathrm{~ms}$.

\section{FUTURE WORKS}

The future activities of this project, at first, will be the design of a real scenario test session in order to explore both: interaction between humans and robots, and robustness of the system architecture. Tests and survey are going to be performed in the future. Usability evaluations will be done by the museum guide who controls the robot in tele-operation with the support of the developed GUI.

After the development of a tablet application for a cultural gaming designed to interact directly with the robot is provided. In a second time, given the potentiality of our CRP, we expect to connect different places of the cultural heritage of "Terra di Savoia" in Piedmont territory through the use of many Virgillike robots in order to create a cultural network between different touristic places and enrich the museum visit experience.

\section{REFERENCES}

[1]. I. Nourbakhsh, "Robot Futures", Massachusetts Institute of Technology press, Cambridge (U.S.A.), 2013.

[2]. K. Walker, Phd thesis in: "Designing for meaning making in museums. Visitor-constructed trails using mobile digital technologies", London Knowledge Lab Faculty of Culture and Pedagogy Institute of Education, University of London, 2010.

[3]. G. Vitale, "Design di sistema per le istituzioni culturali", Zanichelli, Bologna (Italy), 2013.

[4]. E. Guizzo, "Cloud Robotics: Connected to the cloud, Robot get smarter". "http://spectrum.ieee.org/automaton/robotics/roboticssoftware/cloud robotics", 2011.

[5]. G. Ermacora, A. Toma, S. Rosa, "Fly4SmartCity: a Cloud Robotics Service for Smart City applications", submitted at Journal of Ambient Intelligent and Smart Environments.

[6]. S. Rosa, L.O Russo, B. Bona, "Towards A ROS-Based Autonomous Cloud Robotics Platform for Data Centre", in proceeding of 19th IEEE

International Conference on Emerging Technologies and Factory Automation (ETFA), Barcelona, Spain, 2014. 